The Astrophysical Journal, 593:L65-L68, 2003 August 20

(C) 2003. The American Astronomical Society. All rights reserved. Printed in U.S.A.

\title{
A MASSIVE X-RAY OUTFLOW FROM THE QUASAR PDS 456
}

\author{
J. N. ReEves, ${ }^{1,2,3}$ P. T. O'BRIEN, ${ }^{3}$ AND M. J. WARD ${ }^{3}$ \\ Received 2003 June 5; accepted 2003 July 7; published 2003 July 18
}

\begin{abstract}
We report on XMM-Newton spectroscopic observations of the luminous radio-quiet quasar PDS 456. The hard X-ray spectrum of PDS 456 shows a deep absorption trough (constituting $50 \%$ of the continuum) at energies above $7 \mathrm{keV}$ in the quasar rest frame, which can be attributed to a series of blueshifted K-shell absorption edges due to highly ionized iron. The higher resolution soft X-ray Reflection Grating Spectrometer spectrum exhibits a broad absorption line feature near $1 \mathrm{keV}$, which can be modeled by a blend of L-shell transitions from highly ionized iron (Fe XVII-Fe XXIV). An extreme outflow velocity of $\sim 50,000 \mathrm{~km} \mathrm{~s}^{-1}$ is required to model the K-and L-shell iron absorption present in the XMM-Newton data. Overall, a large column density $\left(N_{\mathrm{H}}=5 \times 10^{23} \mathrm{~cm}^{-2}\right)$ of highly ionized gas $(\log \xi=2.5)$ is required in PDS 456. A high-mass outflow rate of $\sim 10 M_{\odot} \mathrm{yr}^{-1}$ (assuming a conservative outflow covering factor of $0.1 \mathrm{sr}$ ) is derived, which is of the same order as the overall mass accretion rate in PDS 456. The kinetic energy of the outflow represents a substantial fraction $(\sim 10 \%)$ of the quasar energy budget, while the large column and outflow velocity place PDS 456 toward the extreme end of the broad absorption line quasar population.
\end{abstract}

Subject headings: galaxies: active — quasars: individual (PDS 456) — X-rays: galaxies

\section{INTRODUCTION}

PDS 456 is a luminous low-redshift $(z=0.184)$ radio-quiet quasar identified in 1997 (Torres et al. 1997). The optical and infrared spectra (Simpson et al. 1999) show broad Balmer and Paschen lines (e.g., H $\beta$ FWHM $=3000 \mathrm{~km} \mathrm{~s}^{-1}$ ), strong Fe II, a hard (dereddened) optical continuum $\left(f_{\nu} \propto \nu^{-0.1 \pm 0.1}\right)$, and one of the strongest "big blue bumps" of any active galactic nucleus (AGN; Simpson et al. 1999; Reeves et al. 2000).

PDS 456 has a dereddened absolute blue magnitude of $M_{B} \approx-27$ (Simpson et al. 1999), making it at least as luminous as the radio-loud quasar $3 \mathrm{C} 273$. Indeed, PDS 456 is the most luminous known AGN in the local universe $(z<0.3)$, its luminosity being more typical of quasars at $z=2-3$, at the peak of the quasar luminosity function.

PDS 456 was first detected as the X-ray source RXS J172819.3-141600 in the ROSAT All Sky Survey (Voges et al. 1999). Subsequent ASCA, Rossi X-Ray Timing Explorer (RXTE), and BeppoSAX observations showed that it was highly X-ray variable (Reeves et al. 2000, 2002), requiring that the $\mathrm{X}$-ray source was very compact (less than 3 Schwarzschild radii) and accreting with an unusually high efficiency (between $6 \%$ and $41 \%$ ), close to the limit for a rotating Kerr black hole (Thorne 1974). The X-ray spectrum of PDS 456, obtained by $A S C A$ and $R X T E$, also appeared to be unusual for a quasar. A strong edgelike feature was present in the iron K-shell band, due to either a highly ionized absorber or reflector, while absorption toward the soft X-ray band was also observed (Reeves et al. 2000). An XMM-Newton observation of PDS 456 was subsequently obtained in AO-1, in order to obtain high signalto-noise spectra of both the iron K-shell complex with the European Photon Imaging Camera (EPIC) CCD detectors and a high-resolution spectrum of the soft X-ray absorber with the XMM-Newton Reflection Grating Spectrometer (RGS). Here we report on the X-ray spectra obtained through the $X M M$ -

\footnotetext{
${ }^{1}$ Laboratory for High Energy Astrophysics, NASA Goddard Space Flight Center, Code 662, Greenbelt Road, Greenbelt, MD 20771; jnr@milkyway .gsfc.nasa.gov.

${ }^{2}$ Universities Space Research Association.

${ }^{3} \mathrm{X}$-Ray and Observational Astronomy Group, University of Leicester, Leicester LE1 7RH, UK.
}

Newton observations of PDS 456, which reveal evidence for a massive outflow, with a velocity of $\sim 50,000 \mathrm{~km} \mathrm{~s}^{-1}$. A previous paper (Reeves et al. 2002) discussed in detail the X-ray variability during the $X M M-N e w t o n$ observation and a simultaneous week-long BeppoSAX observation.

\section{THE X-RAY OBSERVATIONS}

PDS 456 was observed by XMM-Newton on 2001 February 26 with a duration of $40 \mathrm{ks}$. Data were taken with the EPICpn detector (Struder et al. 2001) in full window mode and with the EPIC-MOS detector (Turner et al. 2001) in large window mode. The data were reduced using version 5.4 of the XMMSAS software. Note that the time-averaged $0.5-10 \mathrm{keV}$ flux of PDS 456 during the observation was $9.7 \times 10^{-12} \mathrm{ergs} \mathrm{cm}^{-2} \mathrm{~s}^{-1}$; at this flux level the effect of photon pileup is small $(<1 \%)$. All time intervals were included, as the observation contained no background flares. Data were selected using event patterns 0-12 (for the MOS) and patterns 0-4 (for the pn), and only good Xray events were included. The spectra were extracted from circular source regions of $40^{\prime \prime}$ radius, while background spectra were extracted from an offset circle, close to PDS 456, but free of any background sources. Response matrices and ancillary response files were generated using the SAS tasks RMFGEN and ARFGEN, respectively.

Background-subtracted spectra were fitted using XSPEC version 11.2, including data over the energy ranges $0.4-12 \mathrm{keV}$ for the EPIC-pn and 0.4-10 keV for the co-added EPIC-MOS spectrum. A Galactic absorption column of $N_{\mathrm{H}}=2 \times 10^{21} \mathrm{~cm}^{-2}$ (Dickey \& Lockman 1990) was included in all the fits. A single absorbed power-law fit (photon index $\Gamma=2.0$ ) to both MOS and pn data is clearly inadequate (reduced $\chi^{2}=10$ ). Figure 1 shows the data/model ratio to this power law; a strong soft excess is observed below $1 \mathrm{keV}$ and a large drop in counts is observed above $7 \mathrm{keV}$ in the iron $\mathrm{K}$-shell band. The MOS and pn spectra are consistent, except below $0.5 \mathrm{keV}$, where there is a small divergence in spectral slope. 


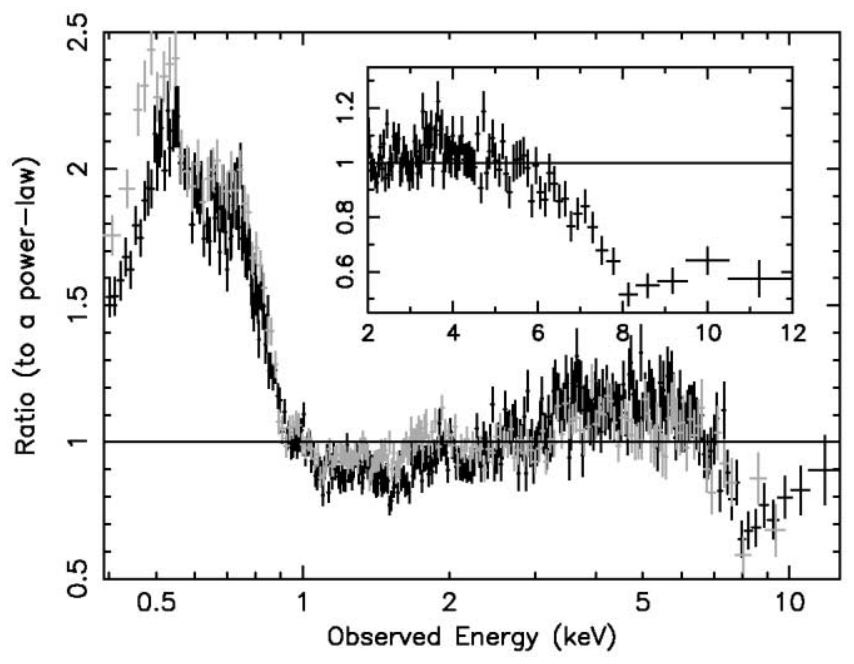

FIG. 1.-Ratio of the broadband X-ray spectrum of PDS 456 to a simple Galactic absorbed power-law model (with $\Gamma=2.0$ ). EPIC-pn data are plotted in black, EPIC-MOS in gray. A clear soft X-ray excess is observed below $1 \mathrm{keV}$, as well as a large decrease in counts above $7 \mathrm{keV}$. The inset plots the data/model ratio to a power-law fit to the $2-12 \mathrm{keV}$ EPIC-pn spectrum in greater detail. A 50\% drop in counts above the iron K-shell band is observed. Note that the energy scale is plotted in units of $\mathrm{keV}$ in the observed frame.

\section{THE IONIZED ABSORBER IN PDS 456}

Initially, to model the iron K-shell band, the data were fitted from 2-12 keV (or 2-10 keV for EPIC-MOS), so that the effects of the large soft excess are negligible. As the EPIC-pn data contained significantly more counts than the EPIC-MOS at the highest energies, we proceeded to fit the EPIC-pn data only. The ratio spectrum above $2 \mathrm{keV}$ to a power law fitted over the $2-5 \mathrm{keV}$ range (i.e., outside of the iron $K$ band) is shown in the inset to Figure 1. A strong deficit of counts occurs above $7 \mathrm{keV}$, where one would expect to observe K-shell absorption edges from highly ionized iron. Indeed, a simple Galactic absorbed power law produced a poor fit $\left(\chi^{2} /\right.$ degrees of freedom $[$ dof] $=1001 / 746$; null hypothesis probability $9 \times$ $\left.10^{-10}\right)$.

Adding three absorption edges between 7 and $10 \mathrm{keV}$ significantly improves the fit $\left(\chi^{2} / \mathrm{dof}=739 / 740\right)$, modeling the broad Fe absorption feature present in the X-ray spectrum. The energies (in the quasar rest frame) and optical depths of the edges are then $E=9.3 \pm 0.1 \mathrm{keV}, E=8.5 \pm 0.2 \mathrm{keV}$, and $E=7.6 \pm 0.3 \mathrm{keV}$ with $\tau=0.56 \pm 0.11, \tau=0.26 \pm 0.11$, and $\tau=0.24 \pm 0.08$, respectively. The breadth of the absorption feature suggests that a wide range of ionizations may be present, while the depth of the feature implies a column of gas of $N_{\mathrm{H}} \sim 10^{24} \mathrm{~cm}^{-2}$. Note that the upper limit to a narrow (i.e., unresolved) $\mathrm{Fe}$ emission line (in the energy range 6.4$6.97 \mathrm{keV}$ ) is less than $12 \mathrm{eV}$, implying that the solid angle subtended by the absorber must be small $(\Omega<0.5 \mathrm{sr})$. Note that an extreme broad iron $\mathrm{K} \alpha$ emission line from an accretion disk cannot model the shape of the spectrum from 2 to $12 \mathrm{keV}$. If one attempts to model the spectrum with a Fe line profile from a rapidly rotating Kerr black hole (Laor 1991), the physical parameters required are unphysical (disk emissivity $R^{-10}$, inclination $=90^{\circ}$, equivalent width $>1 \mathrm{keV}$ ), because the line is (unsuccessfully) attempting to model the broad absorption dip above $7 \mathrm{keV}$ with the blue wing of the line profile.

To obtain a more physical representation of the Fe K-shell absorber, we used a grid of models generated by the XSTAR photoionization code (Kallman et al. 1996) to fit the EPIC-pn
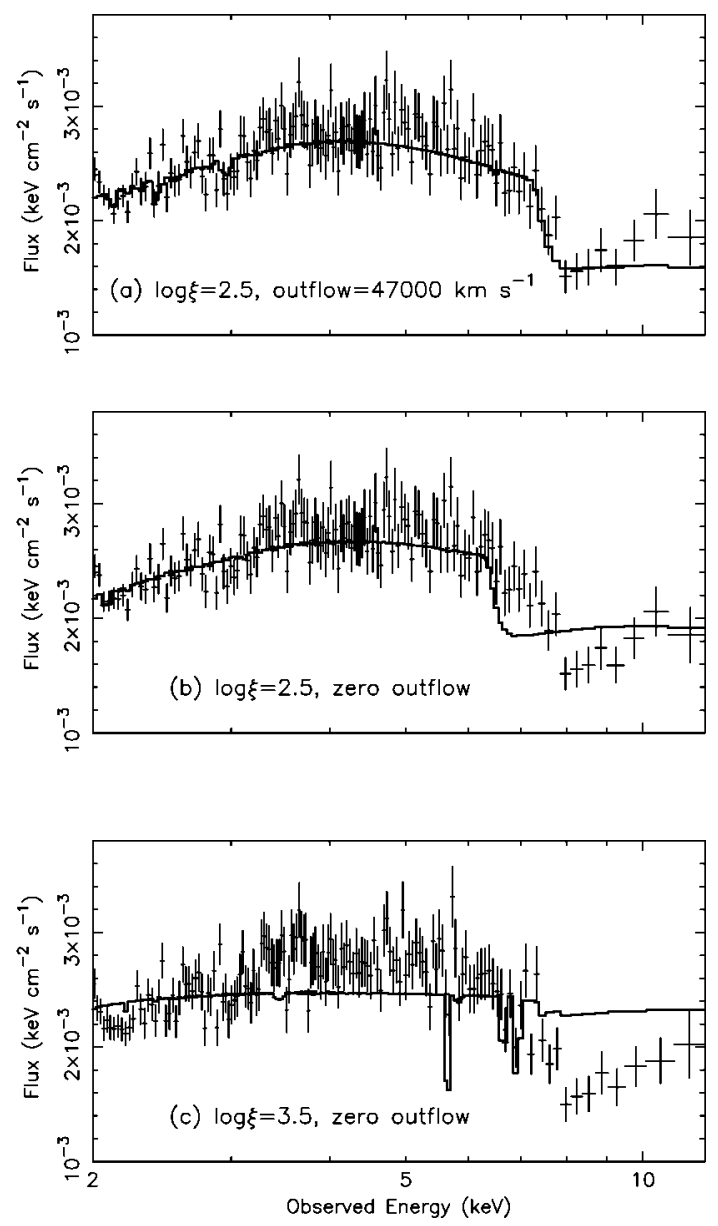

FIG. 2.-Warm absorber fits to the EPIC-pn data of PDS 456 using the XSTAR photoionization code. (a) The best-fit model (solid line) fitted to the EPIC-pn data (plus signs), which requires an outflow velocity of $\sim 50,000 \mathrm{~km} \mathrm{~s}^{-1}$ and an ionization parameter of $\log \xi=2.5$; (b) the same model, but with no outflow velocity, resulting in a significantly worse fit; $(c)$ the same model, but with no outflow and a higher ionization parameter of $\log \xi=3.5$. At this high ionization, the model cannot reproduce the shape of the iron K-shell absorption seen in PDS 456

data above $2 \mathrm{keV}$. Solar elemental abundances were assumed, while a turbulence velocity of $1000 \mathrm{~km} \mathrm{~s}^{-1}$ was used. A one zone photoionization model was adopted, with the column density $\left(N_{\mathrm{H}}\right)$, ionization parameter $(\xi)$, partial covering fraction $(f)$, and outflow velocity $\left(v_{\text {out }}\right)$ as free parameters. An effective hydrogen column density (for a solar abundance $\mathrm{H} / \mathrm{Fe}$ ratio) of $5.7_{-2.5}^{+2.0} \times 10^{23} \mathrm{~cm}^{-2}$ is required, together with an ionization parameter of $\log \xi=2.5 \pm 0.3$ and a partial covering fraction of $f \sim 0.6$, while the power-law continuum steepens to $\Gamma=$ $2.40 \pm 0.05$. At this ionization state, most of the absorption is due to Fe XVII-Fe XXIV (i.e., where the L shell is partially filled). The best-fit spectrum modeled by XSTAR is shown in Figure $2 a$, the depth and energy of the absorption feature being well matched, while the partial covering warm absorber also models the convex spectral curvature (through the increase in opacity at lower energies due to iron L-shell absorption) present in the EPIC-pn data between 2 and $6 \mathrm{keV}$. Overall, the fit statistic for this model is excellent $\left(\chi^{2} / \mathrm{dof}=717 / 740\right)$.

Importantly, a large outflow velocity of $47,000 \mathrm{~km} \mathrm{~s}^{-1}(90 \%$ confidence limits, $36,000-82,000 \mathrm{~km} \mathrm{~s}^{-1}$ ) is needed to model the spectrum. Although the exact outflow velocity is not well constrained by the EPIC-pn data alone, a significant outflow velocity is still required. Figure $2 b$ illustrates this point; if one 
fixes the absorber velocity to zero, then the model fit becomes poor, as the iron $\mathrm{K}$ edges then lie at a significantly lower energy than is observed in the EPIC data. Indeed, if one tries to increase the energy of the model absorption feature by increasing the ionization state of the absorber (so that He- and H-like iron dominates), the result is a worse fit still, partly because the absorption edge feature becomes weaker (as a significant fraction of iron becomes fully ionized) and also because the most prominent absorption features are now the strong $\mathrm{K} \alpha$ and $\mathrm{K} \beta$ absorption lines due to Fe XXVI, which are not present in the actual XMM-Newton data (Fig. 2c). Thus a significant outflow velocity is required by the data; indeed, one can place a conservative lower limit (at $99.9 \%$ confidence) on the velocity of $24,000 \mathrm{~km} \mathrm{~s}^{-1}$ ( or $\sim 0.08 \mathrm{c}$ ).

\subsection{The Soft X-Ray RGS Spectrum of PDS 456}

Extrapolation of the hard X-ray spectrum to lower energies (down to $0.4 \mathrm{keV}$ ) leaves clear residuals in the EPIC-MOS and pn data (e.g., Fig. 1). A strong soft excess is present below $1 \mathrm{keV}$, while residuals due to an additional absorber may also be present in the soft X-ray band. To investigate this at higher spectral resolution, we used data available from the RGS below $2 \mathrm{keV}$. The most noticeable feature present is a broad absorption trough observed between 12 and $15 \AA$ (see Fig. 3). The feature is highly significant, being detected at greater than $99.99 \%$ confidence. When parameterized by a simple broad Gaussianshaped absorption line, the best-fit parameters (in the quasar rest frame) are $E=1.07 \pm 0.02 \mathrm{keV}$ with an equivalent width of $26 \pm 10 \mathrm{eV}$ and a velocity width of $9000 \pm 3000 \mathrm{~km} \mathrm{~s}^{-1}$.

As in the previous section, we used our grid of photoionization models to characterize the properties of the absorbing gas, apparent in the RGS spectrum. A lower column density $\left(N_{\mathrm{H}} \sim 5 \times 10^{22} \mathrm{~cm}^{-2}\right)$ is required to fit the data, while both a similar ionization parameter $(\log \xi=2.6 \pm 0.5)$ and outflow velocity (with $v_{\text {out }}=57,000_{-10,000}^{+8000} \mathrm{~km} \mathrm{~s}^{-1}$ ) are needed, as per the fits to the EPIC-pn data. Partial covering is not required in the RGS fits. The photon index obtained $(\Gamma=2.4$, after correcting for absorption) is consistent with the EPIC-pn data, while a soft blackbody component (with $k T_{\mathrm{BB}}=80 \pm 6 \mathrm{eV}$ ) is also needed in the fit. The lower column density derived from the RGS fit indicates that the absorber is patchy and thus is likely to be located quite close to the X-ray source. Thus we see a higher (partially covered) column along the line of sight toward the hard X-ray continuum, while the column toward the soft X-ray continuum is lower.

Inspection of the best-fit photoionization model implies that a blend of L-shell transitions from Fe XVII to Fe XXIV as well as K-shell absorption from $\mathrm{Ne}$ IX to $\mathrm{Ne} \mathrm{X}$ is responsible for the broad absorption feature at $12-15 \AA$. A weak Ly $\alpha$ absorption line due to O VIII may also be present at $19 \AA$. The outflow velocity derived appears robust, because of the positioning of the broad absorption feature. If one fixes the absorber velocity at zero in the quasar rest frame, then the Fe L-shell absorption in the model is located at energies below the observed absorption trough, while increasing the ionization of the absorber only serves to significantly weaken the soft X-ray absorber.

\section{DISCUSSION}

X-ray spectroscopy of PDS 456 with XMM-Newton has revealed a high column density $\left(N_{\mathrm{H}}=5 \times 10^{23} \mathrm{~cm}^{-2}\right)$ highly ionized $(\log \xi=2.5)$ warm absorber, its main signature being a series of K- and L-shell absorption edges and lines due to iron in the ionization range Fe XVII-Fe XXIV. Crucially, a near-

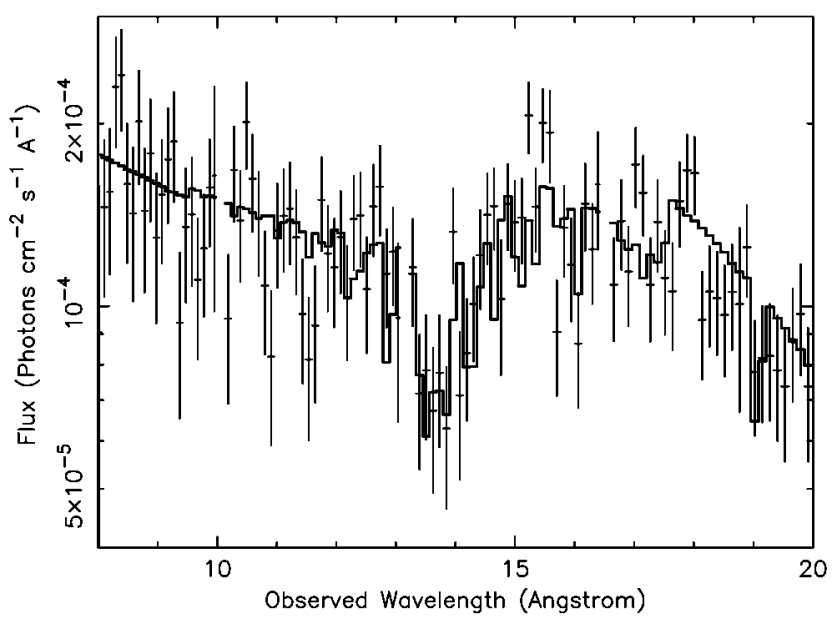

FIG. 3.-XMM-Newton RGS-2 spectrum of PDS 456; the data points are shown as plus signs. A strong absorption trough is present at $12-15 \AA$, observed frame. The best-fit photoionization model (solid line) is superimposed on top of the data points; a high outflow velocity of $\sim 50,000 \mathrm{~km} \mathrm{~s}^{-1}$ is required for the absorber. Note that because of a malfunctioning chip, RGS-1 does not containing data in the 12-15 $\AA$ range and thus is not plotted. Most of the opacity of the absorber results from L-shell transitions of iron in the ionization range Fe XVII-Fe XXIV.

relativistic outflow velocity is required, of the order of 50,000 $\mathrm{km} \mathrm{s}^{-1}$. This velocity is similar to the actual systemic velocity of PDS 456 (at $z=0.184$ ); therefore, it is possible that the absorbing material is unrelated to the quasar and is local to our Galaxy. However, we consider it extremely unlikely that such a high-column highly ionized absorber could occur by chance along the local line of sight. Furthermore, although the Galactic column toward PDS 456 is moderately high $\left(N_{\mathrm{H}}=2 \times 10^{21}\right.$ $\mathrm{cm}^{-2}$ ), this neutral absorption does not contribute toward the features seen in the iron K- and L-shell bands and, in any event, has been accounted for in all our modeling. Therefore it is highly probable that the absorber is intrinsic to the quasar PDS 456.

\subsection{A Massive Outflow in PDS 456}

The combination of a large column density, high ionization, and a fast outflow velocity in PDS 456 indicates that the absorber is located close to the quasar's central engine and that its mass outflow rate is significant relative to the accretion rate. A maximum distance to the absorber can be calculated on the condition that its thickness $(\Delta R)$ cannot exceed its distance from the ionizing source $(R)$, i.e., $\Delta R / R<1$. This requires that $R<10^{4} R_{g}$ (where $R_{g}=G M / c^{2}$ ) for a black hole mass of $10^{9} M_{\odot}$ for PDS 456 (Reeves et al. 2000); thus, it is located within the broad-line region. One likely possibility is that the absorber arises through a disk-driven outflow or wind. The exact origin of these winds and the mechanism responsible for driving them (radiation and/or magnetic) is unclear. For broad absorption line (BAL) quasi-stellar objects, line-driven winds have been proposed (Murray et al. 1995; Proga, Stone, \& Kallman 2000) as well as centrifugal driving along open magnetic field lines (Bottorff et al. 1997).

Using conservation of mass, the outflow rate $\left(\dot{M}_{\text {out }}\right)$ for an outflow subtending a solid angle of $\Omega$ steradian with a constant velocity $v_{\text {out }}$ is given by $\dot{M}_{\text {out }}=\Omega n r^{2} v_{\text {out }} m_{p}$, where $n$ is the density, $r$ the distance from the X-ray source, and $m_{p}$ the proton mass. Now the ionization parameter $\xi$ is defined as $\xi=$ $L_{\mathrm{X}} / n r^{2}$, so we can obtain $\dot{M}_{\text {out }}=\Omega v_{\text {out }} m_{p} L_{\mathrm{X}} / \xi$.

From the observations, the hard X-ray luminosity of PDS 
456 (above $2 \mathrm{keV}$ ) is $\sim 10^{45} \mathrm{ergs} \mathrm{s}^{-1}$ and the ionization parameter is $\xi \sim 1000$, while $v=50,000 \mathrm{~km} \mathrm{~s}^{-1}$. From the lack of any iron $\mathrm{K}$ line emission it is likely that the outflow subtends a solid angle of less than 0.5 sr. However, even for a conservative value of $0.1 \mathrm{sr}$, the mass outflow rate derived is $\dot{M}_{\text {out }}=8 \times 10^{26} \mathrm{~g} \mathrm{~s}^{-1}$ or $\sim 10 M_{\odot} \mathrm{yr}^{-1}$. A lower limit of $\sim 5 M_{\odot} \mathrm{yr}^{-1}$ is obtained using the $99.9 \%$ confidence lower bound on the outflow velocity of $v_{\text {out }}=24,000 \mathrm{~km} \mathrm{~s}^{-1}$. Note that the outflow rate calculated assumes that the gas is in equilibrium; if the gas is being accelerated then the actual rate may differ.

PDS 456 is an extremely luminous quasar with a bolometric luminosity of $L_{\mathrm{bol}} \sim 10^{47} \mathrm{ergs} \mathrm{s}^{-1}$ and a black hole mass of $10^{9} M_{\odot}$, assuming that accretion occurs near the Eddington limit (Simpson et al. 1999; Reeves et al. 2000). The mass accretion rate for PDS 456 , given by $\dot{M}=L_{\mathrm{bol}} / \eta c^{2}$, is $\sim 20 M_{\odot}$ $\mathrm{yr}^{-1}$, for an efficiency of $\eta=0.1$. Thus it seems likely that the outflow rate is of the same order as the mass accretion rate in PDS 456. Similarly, the kinetic output of the outflow in PDS 456 is large, about $10^{46} \mathrm{ergs} \mathrm{s}^{-1}$, corresponding to $10 \%$ of the bolometric output of the quasar. The high velocity also implies that the outflow is likely to be driven from the accretion disk at a fairly small radius. For a Keplerian disk, the launch radius for the outflow is simply $R_{\text {out }}=c^{2} / v_{\text {esc }}^{2} R_{g}$, where $v_{\text {esc }}$ is the escape velocity of the material. Assuming that $v_{\text {esc }} \sim v_{\text {out }}$ (although some acceleration of the matter will probably occur), this implies that the outflow in PDS 456 is launched from a radius of $\sim 40 R_{g}$ (about $10^{16} \mathrm{~cm}$ for a $10^{9} M_{\odot}$ black hole). Indeed, the launch radius may be smaller if the gas is not in simple equilibrium.

PDS 456 appears to be in a small but growing group of AGNs to show extreme absorption properties in the iron K-shell band. The lensed BAL quasar APM $08279+5255$ has been found to exhibit iron K absorption lines and/or edges (Chartas et al. 2002; Hasinger, Schartel, \& Komossa 2002) in both Chandra and XMM-Newton observations. A similar absorber has also been reported in the BAL quasar PG 1115+080 (Chartas, Brandt, \& Gallagher 2003). The outflow velocities are similar to PDS 456, in the range $(0.1-0.4) c$. Given this apparent association between high-velocity X-ray outflowing gas and the UV BALs, one may predict to see BAL features in the UV spectrum of PDS 456. Indeed, a Hubble Space Telescope Space Telescope Imaging Spectrograph spectrum of PDS 456 (P. T. O'Brien et al. 2003, in preparation) reveals that there may be cooler gas in the outflow; a BAL is seen in $\mathrm{Ly} \alpha$ with an outflow velocity of
$12,000-22,000 \mathrm{~km} \mathrm{~s}^{-1}$, while the $\mathrm{C}$ IV emission line is also blueshifted by $\sim 5000 \mathrm{~km} \mathrm{~s}^{-1}$. Thus PDS 456 may reside just at the extreme end of the BAL quasar population (Turnshek et al. 1988; Weymann et al. 1991).

Similar highly ionized X-ray outflows have recently been discovered in the non-BAL radio-quiet quasars PG 1211+143 and PG 0844+349 (Pounds et al. 2003a, 2003b), suggesting that these properties are not unique to UV-identified BAL quasars. We also note that strong iron $\mathrm{K}$ absorption has also been observed in the narrow-line Seyfert 1 galaxies 1H 0707-495 and IRAS 13324-3809 (Boller et al. 2002, 2003) and also in some X-ray binaries, e.g., GRS 1915+105 (Lee et al. 2002), GX 13+1 (Sidoli et al. 2002), and X 1624-490 (Parmar et al. 2002). In the quasars PDS 456, PG $1211+143$, and PG $0844+349$, the X-ray columns are near to $10^{24} \mathrm{~cm}^{-2}$, the outflow velocities measured are $\sim 0.1 c$, while the ionizations are also $\operatorname{similar}\left(\xi \sim 10^{3}\right)$. Using PG $1211+143$ as an example, King \& Pounds (2003) have recently demonstrated, for radiatively driven outflows, that objects accreting near to the Eddington rate are likely to contain massive $\left(\dot{M}_{\text {out }} \sim \dot{M}_{\text {Edd }}\right)$ Compton-thick winds.

Indeed, when the outflow rate is of the same order as the accretion rate, the flow can become optically thick at $\sim(10-100) R_{g}$. This can account for both the high columns and the velocities of the X-ray outflows as well as provide a natural source of thermalized photons for the UV and soft X-ray excesses observed in these quasars.

However, other scenarios are possible; for instance, Reeves et al. (2002) recently used a magnetic flare model to explain the rapid X-ray variability in PDS 456, through coherent flaring events occurring when the accretion rate is close to Eddington. Indeed, it is conceivable that the outflowing matter in PDS 456 is the ejecta associated with these flares. Interestingly, the output of the magnetic events $\left(\sim 10^{46} \mathrm{ergs} \mathrm{s}^{-1}\right)$ in PDS 456 is very similar to the kinetic power of the outflow, so this scenario seems energetically plausible. Overall, the extreme properties of the outflow in PDS 456 seem linked to its high accretion rate (Reeves et al. 2000), which appears to drive the energetics of its unusually massive outflow.

This Letter is based on observations obtained with $X M M$ Newton, an ESA science mission with instruments and contributions directly funded by ESA Member States and the US (NASA). We thank Ken Pounds and Andrew King for discussions and Kim Page for performing spectral fits. James Reeves thanks the Leverhulme Trust for their support.

\section{REFERENCES}

Boller, Th., Tanaka, Y., Fabian, A. C., Brandt, W. N., Gallo, L., Anabuki, N., Haba, Y., \& Vaughan, S. 2003, MNRAS, in press (astro-ph/0305239)

Boller, Th., et al. 2002, MNRAS, 329, L1

Bottorff, M. C., Korista, K. T., Shlosman, I., \& Blandford, R. D. 1997, ApJ, 479, 200

Chartas, G., Brandt, W. N., \& Gallagher, S. C. 2003, ApJ, in press (astro-ph/ 0306125)

Chartas, G., Brandt, W. N., Gallagher, S. C., \& Garmire, G. P. 2002, ApJ, 579,169

Dickey, J. M., \& Lockman, F. J. 1990, ARA\&A, 28, 215

Hasinger, G., Schartel, N., \& Komossa, S. 2002, ApJ, 573, L77

Kallman, T., Liedahl, D., Osterheld, A., Goldstein, W., \& Kahn, S. 1996, ApJ, 465, 994

King, A., \& Pounds, K. A. 2003, MNRAS, in press (astro-ph/0305541)

Laor, A. 1991, ApJ, 376, L90

Lee, J., Reynolds, C. S., Remillard, R., Schultz, N., Blackman, E. G., \& Fabian, A. C. 2002, ApJ, 567, 1102

Murray, N., Chiang, J., Grossman, S. A., \& Voit, G. M. 1995, ApJ, 451, 498

Parmar, A., Oosterbroek, T., Boirin, L., \& Lumb, D. 2002, A\&A, 386, 910

Pounds, K. A., King, A., Page, K., \& O'Brien, P. T. 2003a, MNRAS, submitted (astro-ph/0305571)
Pounds, K. A., Reeves, J. N., King, A., Page, K., \& O’Brien, P. T. 2003b, MNRAS, in press (astro-ph/0303603)

Proga, D., Stone, J. M., \& Kallman, T. 2000, ApJ, 543, 686

Reeves, J. N., O'Brien, P. T., Vaughan, S., Law-Green, D., Ward, M., Simpson, C., Pounds, K. A., \& Edelson, R. 2000, MNRAS, 312, L17

Reeves, J. N., Wynn, G., O'Brien, P. T., \& Pounds, K. A. 2002, MNRAS, 336, L56

Sidoli, L., Parmar, A., Oosterbroek, T., \& Lumb, D. 2002, A\&A, 385, 940

Simpson, C., Ward, M., O’Brien, P. T., \& Reeves, J. N. 1999, MNRAS, 303, L23

Strüder, L., et al. 2001, A\&A, 365, L18

Thorne, K. S. 1974, ApJ, 191, 507

Torres, C. A. O., et al. 1997, ApJ, 488, L19

Turner, M. J. L., et al. 2001, A\&A, 365, L27

Turnshek, D. A., Grillmair, C. J., Foltz, C. B., \& Weymann, R. J. 1988, ApJ, 325,651

Voges, W., et al. 1999, A\&A, 349, 389

Weymann, R. J., Morris, S. L., Foltz, C. B., \& Hewett, P. C. 1991, ApJ, 373, 23 